\title{
Diffusion in disordered lattices and related Heisenberg ferromagnets
}

\author{
M. D. Grynberg ${ }^{1}$ and R. B. Stinchcombe ${ }^{2}$ \\ ${ }^{1}$ Departamento de Física, Universidad Nacional de La Plata, C.C. 67, (1900) La Plata, Argentina \\ ${ }^{2}$ Theoretical Physics, Department of Physics, University of Oxford, 1 Keble Road, Oxford OX1 3NP, United Kingdom
}

(Received 3 August 1999)

\begin{abstract}
We study the diffusion of classical hard-core particles in disordered lattices within the formalism of a quantum spin representation. This analogy enables an exact treatment of noninstantaneous correlation functions at finite particle densities in terms of single spin excitations in disordered ferromagnetic backgrounds. Applications to diluted chains and percolation clusters are discussed. It is found that density fluctuations in the former exhibit a stretched exponential decay while an anomalous power law asymptotic decay is conjectured for the latter.

PACS number(s): 02.50.- r, 75.10.Jm, 82.20.Mj, 05.50.+q
\end{abstract}

\section{INTRODUCTION}

Processes involving classical particles constrained to diffuse and interact stochastically on discrete substrates is one of the oldest schemes for the study of a rich variety of relaxation phenomena [1-3]. Although from a conceptual standpoint such processes give only a phenomenological level of understanding, they do provide the type of behavior necessary to describe irreversibility in many-body systems which otherwise, would be difficult to derive from first principles. Among the main techniques to obtain the time probability distribution of these processes, possibly the master equation (MEQ) approach [4] is the more directly related to physical concepts. Despite its apparent simplicity however, it generally gives rise to an infinite hierarchy of coupled equations whose solutions become quite involved to elucidate, particularly at large times. In a concerted effort to remedy this situation, quantum field theory methods have recently regained new impetus in the study of the dynamics of non-quantummechanical objects [5]. Basically, the underlying idea is that the MEQ resembles a time-dependent Schrödinger equation in a pure imaginary time with probability distributions playing the role of wave functions. Thus, by studying the field theory associated to the MEQ a formal solution, in some cases exact, of many-body probability distributions can be found explicitly [6].

As a contribution to the current momentum of this approach, in this work we shall revisit the problem of hard-core particles diffusing in quenched disordered lattices emphasizing the formal analogy with its quantum counterpart. The avoidance of double occupancy accounts for the essential aspects of both interparticle interactions and mobility whereas frozen-in disordered bonds $\left\{J_{\mathbf{r}, \mathbf{r}^{\prime}}\right\}$, modeling random hopping rates between locations $\left\{\mathbf{r}, \mathbf{r}^{\prime}\right\}$, is essential to understand the slowing down of transport properties in a vast family of inhomogeneous and glassy systems $[2,3,7]$.

Following the thread of ideas initiated in Ref. [8] and developed subsequently by many authors in related processes $[5,9]$, here we attempt to further advance the subject forward by means of a (pseudo) spin- $\frac{1}{2}$ description in which spin up or down at a given site corresponds to particle or vacancy, say, at that location. The MEQ is then equivalent to the action of a quantum spin "Hamiltonian" encompassing the original transition probability rates. Interestingly, for the case of symmetric diffusion of hard core particles the resulting Hamiltonian reduces to an isotropic Heisenberg ferromagnet whose full rotational symmetry applies irrespective of the hopping disorder. Although this continuous symmetry is ultimately imposed by conservation of probability throughout the Brownian process, its exploitation is not evident without appealing to the quantum spin Hamiltonian analogy [10]. Particularly, this formalism becomes advantageous in analyzing spontaneous fluctuations of the steady state such as noninstantaneous density-density correlation functions (e.g., structure factors and scattering functions). As we shall see, the use of elementary quantum mechanical considerations along with selection rules based on the conservation of total angular momentum enable an exact treatment of these manybody correlations in terms of single spin excitations.

The layout of the paper is organized as follows. In Sec. II, we recast the MEQ of the disordered Brownian process as a physical realization of the Heisenberg ferromagnet. Sections II A and II B treat in turn the structure of the steady state and its noninstantaneous two-point correlations. Section III discusses autocorrelation functions in a variety of scenarios. An exact treatment of these functions in diluted chains is given in Sec. III A, where a stretched exponential asymptotic dynamics exhibiting a scaling regime is found. Section III B continues with a discussion of diluted lattices in higher dimensions. Exploiting scaling pictures for the frequency and the density of states of single excitations on percolation clusters at criticality [11], we are led to suggest a slower or anomalous diffusive kinetics. We end the paper with Sec. IV which contains our conclusions, along with some remarks on extensions of the present work.

\section{MASTER EQUATION AND SPIN REPRESENTATION}

A central assumption underlying most phenomenological stochastic models is that the actual nonequilibrium dynamics of real systems can be approximated by a discrete Markovian process and, therefore described by a MEQ. The latter governs entirely the time evolution of the probabilities $P(s, t)$ of finding the system in a certain configuration $|s\rangle$ at time $t$. If $W\left(s \rightarrow s^{\prime}\right)$ denotes the rate or transition probability per unit 
time at which configuration $|s\rangle$ evolves to $\left|s^{\prime}\right\rangle$, the MEQ reads

$$
\partial_{t} P(s, t)=\sum_{s^{\prime}}\left[W\left(s^{\prime} \rightarrow s\right) P\left(s^{\prime}, t\right)-W\left(s \rightarrow s^{\prime}\right) P(s, t)\right] .
$$

Assuming the basis vectors $|s\rangle$ form an orthonormal set, it is useful to describe the ensemble averaged state vector of the system at time $t$ as $|P(t)\rangle=\Sigma_{s} P(s, t)|s\rangle$. Thus, starting from a given probability distribution $|P(0)\rangle$ it can be readily checked that the formal integration of Eq. (1) yields a state vector solution of the type

$$
|P(t)\rangle=e^{-H t}|P(0)\rangle,
$$

where the matrix elements of the transition operator $H$ (or "Hamiltonian") are constructed as [12]

$$
\begin{gathered}
\left\langle s^{\prime}|H| s\right\rangle=-W\left(s \rightarrow s^{\prime}\right), \quad s^{\prime} \neq s, \\
\langle s|H| s\rangle=\sum_{s^{\prime} \neq s} W\left(s \rightarrow s^{\prime}\right) .
\end{gathered}
$$

The steady states of our stochastic processes correspond to the ground states of $H$ of each subspace within which the dynamics takes place, all with the zero eigenvalue. Any eigenvalues with positive real parts $\lambda>0$ correspond to decaying states with lifetime $1 / \lambda$. Due to probability conservation, clearly every column of $H$ adds up to zero, i.e., $\Sigma_{s^{\prime}}\left\langle s^{\prime}|H| s\right\rangle=0$. Thus, in passing and for future reference it is worth pointing out that a left steady state can be immediately obtained as

$$
\langle\tilde{\psi}|=\sum_{s}\langle s|,
$$

which evidently satisfies $\langle\widetilde{\psi}| H=0$. Also, notice that $\langle\widetilde{\psi} \mid P(t)\rangle \equiv 1 \forall t$.

Turning to the analogy between diffusion of hard-core classical particles and quantum spin-1/2 systems we now represent a particle or vacancy, respectively, at site $\mathbf{r}$ by spin up or down, i.e., by $m_{\mathbf{r}}=+1,-1$ where $m_{\mathbf{r}}$ is an eigenvalue of the $z$ component (say) of the Pauli operator $\vec{\sigma}(\mathbf{r})$ for site r. Clearly a typical configuration $|s\rangle$ in a general lattice with $N$ locations can be characterized by a direct product $\left|m_{\mathbf{r}_{1}}, \ldots, m_{\mathbf{r}_{N}}\right\rangle$ of all the site spinors. So the set $\{W(s$ $\left.\left.\rightarrow s^{\prime}\right)\right\}=\left\{J_{\mathrm{rr}}^{\prime}\right\}$ of transition hopping rates between connected sites $\mathbf{r}, \mathbf{r}^{\prime}$ (e.g., nearest neighbors $\left\langle\mathbf{r}, \mathbf{r}^{\prime}\right\rangle$ ), then describes the following spin-exchange process

$$
\begin{aligned}
\mid m_{\mathbf{r}_{1}} \cdots m_{\mathbf{r}} & \left.=1 \cdots m_{\mathbf{r}^{\prime}}=-1 \cdots m_{\mathbf{r}_{N}}\right\rangle \leftrightarrow \mid m_{\mathbf{r}_{1}} \cdots m_{\mathbf{r}} \\
& \left.=-1 \cdots m_{\mathbf{r}^{\prime}}=1 \cdots m_{\mathbf{r}_{N}}\right\rangle .
\end{aligned}
$$

Here and in the following the set $\left\{J_{\mathbf{r r}^{\prime}}\right\}$ can be arbitrarily substitutionally disordered. To construct the associated "Hamiltonian" $H$ whose terms keep proper track of both probability conservation and the spin exchange process, it is useful to cast the discussion in terms of spin raising and lowering operators $\sigma_{\mathbf{r}}^{+}, \sigma_{\mathbf{r}}^{-}$. The off-diagonal part of $H$ exchanging configurations as schematized above, is therefore given by

$$
\sum_{s} \sum_{s^{\prime} \neq s}\left|s^{\prime}\right\rangle\left\langle s^{\prime}|H| s\right\rangle\langle s|=-\sum_{\left\langle\mathbf{r r}^{\prime}\right\rangle} J_{\mathbf{r r}^{\prime}}\left(\sigma_{\mathbf{r}}^{+} \sigma_{\mathbf{r}^{\prime}}^{-}+\text {H.c. }\right),
$$

whereas conservation of probability, i.e., Eq. (4), requires the emergence of a diagonal part of the form

$$
\sum_{s}|s\rangle\langle s|H| s\rangle\langle s|=\sum_{\left\langle\mathbf{r r}^{\prime}\right\rangle} J_{\mathbf{r r}}\left[\hat{n}_{\mathbf{r}}\left(1-\hat{n}_{\mathbf{r}^{\prime}}\right)+\left(1-\hat{n}_{\mathbf{r}}\right) \hat{n}_{\mathbf{r}^{\prime}}\right],
$$

where $\hat{n}_{\mathbf{r}} \equiv \sigma_{\mathrm{r}}^{+} \sigma_{\mathrm{r}}^{-}$denote occupation number operators. These terms basically count the total number of ways in which a given configuration $|s\rangle$ can evolve to different states $\left|s^{\prime}\right\rangle$ through a particle hopping attempt between nearest neighbor pairs $\left\langle\mathbf{r r}^{\prime}\right\rangle$, weighting each accessible attempt with rate $J_{\mathbf{r r}^{\prime}}$. This yields precisely the right hand side of Eq. (4). Thus, in terms of usual spin-1/2 Pauli matrices $\vec{\sigma}$ $\equiv\left(\sigma^{x}, \sigma^{y}, \sigma^{z}\right)$, by virtue of Eqs. (6) and (7) the evolution operator $H$ reduces finally to a Heisenberg ferromagnet

$$
H=-\frac{1}{2} \sum_{\left\langle\mathbf{r r}^{\prime}\right\rangle} J_{\mathbf{r r}}\left(\vec{\sigma}_{\mathbf{r}} \cdot \vec{\sigma}_{\mathbf{r}^{\prime}}-1\right) .
$$

Interestingly, due the isotropic nature of the interactions, the stochastic dynamics leaves invariant all the components of the total angular momentum $\mathbf{S}=\frac{1}{2} \sum_{\mathbf{r}} \vec{\sigma}_{\mathbf{r}}$, namely, $[H, \mathbf{S}]=0$ irrespective of the disordered background of hopping rates $J_{\mathbf{r r}^{\prime}}$. As a consequence of the full rotational symmetry, the calculation of spontaneous density fluctuations are simplified remarkably (Sec. II B). In preparation for the analysis of those functions, let us first examine the form of the steady state at finite particle densities.

\section{A. The steady state}

Evidently, the fully jammed ferromagnetic state $|F\rangle$ $=\left|S, S^{z}\right\rangle$ with total spin $S=N / 2$ and total magnetization $S^{z}$ $=N / 2$ is a steady state $(\mathrm{SS})$. Since $\left[H, S^{-}\right]=0$ we can generate normalized SS $\left|\psi_{m}\right\rangle$ with $\left|S, S^{z}\right\rangle=|N / 2, N / 2-m\rangle$, i.e., having $m$ vacancies and particle density $\rho=1-m / N$, and such that $H\left|\psi_{m}\right\rangle=0$ by applying $m$ times the lowering operator $S^{-}=\Sigma_{\mathbf{r}} \sigma_{\mathbf{r}}^{-}=S^{x}-i S^{y}$, namely,

$$
\left|\psi_{m}\right\rangle=\alpha_{m}\left(S^{-}\right)^{m}|F\rangle,
$$

where the normalization factor is $\alpha_{m}=\sqrt{(N-m) ! / N !}$.

Notice that $\left|\psi_{m}\right\rangle$ is an equally weighted linear combination of all permissible $\Omega=\left(\begin{array}{l}N \\ m\end{array}\right)$ configurations with $(N-m)$ particles. In particular,

$$
\left\langle\psi_{m}\right|=\frac{1}{\sqrt{\Omega}}\langle\widetilde{\psi}|,
$$

which is in line with the more basic observation that, aside from normalization prefactors, left and right SS should coincide since Eq. (8) involves a self-adjoint evolution operator, 
namely, $W\left(s \rightarrow s^{\prime}\right)=W\left(s^{\prime} \rightarrow s\right)$. From this latter observation, it is worth pointing out that detailed balance arises immediately in Eq. (1) given that $P(s) \rightarrow 1 / \Omega \forall|s\rangle$.

The structure of these equilibrium states is rather trivial regardless of the hopping disorder. This follows by noting that

$$
\hat{n}_{\mathbf{p}} \hat{n}_{\mathbf{q}}\left|\psi_{m}\right\rangle=\frac{1}{\sqrt{\Omega}} \sum_{j_{1}<}^{\prime \prime} \cdots \sum_{\sum_{j m}}^{\prime \prime} \sigma_{\mathbf{r}_{j 1}}^{-} \cdots \sigma_{\mathbf{r}_{j m}}^{-}|F\rangle,
$$

where the index ordering denotes sums over all reachable different configurations and the double prime restricts the sums to $\mathbf{r}_{j} \neq \mathbf{p}, \mathbf{q}$. This yields $\left(\begin{array}{c}N-2 \\ m\end{array}\right)$ nonvanishing configurations and therefore

$$
\left\langle\psi_{m}\left|\hat{n}_{\mathbf{p}} \hat{n}_{\mathbf{q}}\right| \psi_{m}\right\rangle=\frac{\left(\begin{array}{c}
N-2 \\
m
\end{array}\right)}{\Omega}=\left(1-\frac{m}{N}\right)\left(1-\frac{m}{N-1}\right)_{\substack{\sim \\
\sim \rightarrow \infty}} \rho^{2},
$$

so density-density correlators decouple in the large system limit. A similar reasoning holds for many-point correlators thus yielding spatially uncorrelated SS. Henceforth, we address our approach to the correlations between fluctuations that occur spontaneously at different times which as is known [13], are closely related to the relaxation dynamics governing nonequilibrium regimes.

\section{B. Noninstantaneous steady state correlations}

We will be especially interested in the calculation of noninstantaneous joint probability distributions $\langle\mathcal{A}(t) \mathcal{B}(0)\rangle$ of quantities $\mathcal{A}, B$ such as local densities or local density correlations, i.e., diagonal operators in the particle or $\sigma^{z}$ representation. Here, the brackets indicate an average over histories up to a to time $t$ starting from a given probability distribution $|P(0)\rangle$. More precisely, this can be expressed in terms of the following discrete path integration

$$
\begin{aligned}
\langle\mathcal{A}(t) \mathcal{B}(0)\rangle= & (\Delta t)^{n-1} \sum_{s_{1}} \ldots \sum_{s_{n}} P\left(s_{1}, 0\right)\left\langle s_{1}|\mathcal{B}| s_{1}\right\rangle W\left(s_{1} \rightarrow s_{2}\right) \\
& \times W\left(s_{2} \rightarrow s_{3}\right) \cdots W\left(s_{n-1} \rightarrow s_{n}\right)\left\langle s_{n}|\mathcal{A}| s_{n}\right\rangle,
\end{aligned}
$$

where the sums run over all possible states $\left|s_{j}\right\rangle$, and $\Delta t W\left(s_{j-1} \rightarrow s_{j}\right)$ denotes the probability of evolving from $\left|s_{j-1}\right\rangle$ to $\left|s_{j}\right\rangle$ in a single elementary time step $\Delta t=t /(n$ -1) [14]. However, by construction [Eqs. (3) and (4)], this latter probability is given by $\left\langle s_{j}|1-\Delta t H| s_{j-1}\right\rangle$. Because $\mathcal{A}, B$ are taken diagonal in the particle representation,

$$
\begin{gathered}
P(s, 0)\langle s|\mathcal{B}| s\rangle=\langle s|\mathcal{B}| P(0)\rangle, \\
\langle s|\mathcal{A}| s\rangle=\langle\tilde{\psi}|\mathcal{A}| s\rangle,
\end{gathered}
$$

so, recalling that $(1-\Delta t H)^{n} \rightarrow e^{-H t}$, in the limit of a large number of steps [14], Eq. (13) yields

$$
\langle\mathcal{A}(t) \mathcal{B}(0)\rangle=\left\langle\tilde{\psi}\left|\mathcal{A} e^{-H t} \mathcal{B}\right| P(0)\right\rangle \text {. }
$$

Since $[H, \mathbf{S}]=0$ for any disorder realization, Eq. (15) allows for a systematic treatment of spontaneous density fluctuations. In terms of the local density fields referred to above, this requires consideration of noninstantaneous particleparticle correlations $C_{\mathbf{r}, \mathbf{r}^{\prime}}(t)=\left\langle\hat{n}_{\mathbf{r}}(t) \hat{n}_{\mathbf{r}^{\prime}}(0)\right\rangle$ occurring in the SS distribution $|P(0)\rangle=\left|\psi_{m}\right\rangle / \sqrt{\Omega}$ with $\rho=1-m / N$. As usual, it is convenient to choose a basis $\left\{|\Lambda\rangle=\left|S, S^{z}, \lambda\right\rangle\right\}$ of common eigenstates of $\mathbf{S}^{2}, S^{z}$ and $H$ with eigenvalues $S(S$ $+1), S^{z}$ and $\lambda$ respectively. Inserting an orthogonalized set of $|\Lambda\rangle$ states in Eq. (15), by virtue of Eq. (10) it follows that in the $m$-down-spin subspace $C_{\mathbf{r}, \mathbf{r}^{\prime}}(t)$ can be written as

$$
C_{\mathbf{r}, \mathbf{r}^{\prime}}(t)=\sum_{\text {levels } \lambda>0} e^{-\lambda t}\left\langle\psi_{m}\left|\hat{n}_{\mathbf{r}}\right| \Lambda\right\rangle\left\langle\Lambda\left|\hat{n}_{\mathbf{r}^{\prime}}\right| \psi_{m}\right\rangle
$$

where we have restricted the sum to eigenstates $|\Lambda\rangle$ different from $\left|\psi_{m}\right\rangle$ (which has vanishing eigenvalue $\lambda=0$ ), to subtract the time independent correlations, i.e. $\left\langle\hat{n}_{\mathbf{r}}\right\rangle\left\langle\hat{n}_{\mathbf{r}^{\prime}}\right\rangle=\rho^{2}$. All eigenvalues $\lambda$ are real and positive definite, as by construction $H$ is a self-adjoint stochastic operator.

Selection rules can now be applied to simplify remarkably the calculation. Noting that the Pauli matrices $\sigma_{\mathbf{r}}^{z}$ are tensors of rank one, since $\hat{n}_{\mathbf{r}}=\left(1+\sigma_{\mathbf{r}}^{z}\right) / 2$, the Wigner-Eckart theorem ensures nonvanishing matrix elements $\left\langle\psi_{m}\left|\hat{n}_{\mathbf{r}}\right| \Lambda\right\rangle$ only if the total spin $S$ of $\left|\psi_{m}\right\rangle$ and $|\Lambda\rangle$ differ by 0 or 1, i.e., $S$ $=N / 2, N / 2-1$; in either case with $S^{z}=N / 2-m$. To identify the relevant $|\Lambda\rangle$ states, we first compute the total spin $S$ of a single spin excitation $\left|\varphi_{\lambda}\right\rangle=\Sigma_{\mathbf{r}} \varphi_{\lambda}(\mathbf{r}) \sigma_{\mathbf{r}}^{-}|F\rangle$. Since $\mathbf{S}^{2}$ $=S^{z} S^{z}+\frac{1}{2}\left(S^{+} S^{-}+S^{-} S^{+}\right)$, it can readily checked that

$$
\mathbf{S}^{2}\left|\varphi_{\lambda}\right\rangle= \begin{cases}\left(\frac{N}{2}-1\right) \frac{N}{2}\left|\varphi_{\lambda}\right\rangle & \text { if } \lambda>0, \\ \frac{N}{2}\left(\frac{N}{2}+1\right)\left|\varphi_{\lambda}\right\rangle & \text { if } \lambda=0,\end{cases}
$$

for which the wave function should satisfy $\Sigma_{\mathbf{r}} \varphi_{\lambda}(\mathbf{r})$ $\equiv \sqrt{N} \delta_{\lambda, 0}$. Therefore, the desired $|\Lambda\rangle$ states giving nonzero matrix elements in Eq. (16) are essentially rotated versions of the above single spin excitation states. Specifically, recalling that the angular momentum algebra imposes

$$
S^{-}\left|S, S^{z}\right\rangle=\sqrt{\left(S+S^{z}\right)\left(S-S^{z}+1\right)}\left|S, S^{z}-1\right\rangle,
$$

we can generate a normalized SS in the $m$-down-spin sector by applying $(m-1)$ times the lowering operator $S^{-}$to the spin excitation $\left|\varphi_{\lambda}\right\rangle$, namely,

$$
|\Lambda\rangle=\beta_{m}\left(S^{-}\right)^{m-1}\left|\varphi_{\lambda}\right\rangle,
$$

where the normalization factor arising from the products generated by each application of $S^{-}$turns out to be

$$
\beta_{m}=\sqrt{\frac{(N-m-1) !}{(N-2) !(m-1) !}}, \quad 1<m<N-1 .
$$

Thus, for $|\Lambda\rangle \neq\left|\psi_{m}\right\rangle$ these are all the linearly independent states contributing to Eq. (16), having total spin $S=(N / 2$ $-1)$, total magnetization $S^{z}=(N / 2-m)$, and $\lambda>0$. So, we are now left with the calculation of $\left\langle\psi_{m}\left|\hat{n}_{\mathbf{r}}\right| \Lambda\right\rangle$. This matrix element is expanded as 


$$
\begin{aligned}
\left\langle\psi_{m}\left|\hat{n}_{\mathbf{r}}\right| \Lambda\right\rangle & =\beta_{m}\left\langle\psi_{m}\left|\hat{n}_{\mathbf{r}}\left(S^{-}\right)^{m-1}\right| \varphi_{\lambda}\right\rangle \\
& =\beta_{m} \sum_{\mathbf{p}} \varphi_{\lambda}(\mathbf{p})\left\langle\psi_{m}\left|\hat{n}_{\mathbf{r}}\left(S^{-}\right)^{m-1} \sigma_{\mathbf{p}}^{-}\right| F\right\rangle .
\end{aligned}
$$

To go a further step in our analysis notice that

$$
\begin{aligned}
\hat{n}_{\mathbf{r}}\left(S^{-}\right)^{m-1} \sigma_{\mathbf{p}}^{-}|F\rangle= & (m-1) !\left(1-\delta_{\mathbf{r}, \mathbf{p}}\right) \\
& \times \sum_{j_{1}<}^{\prime \prime} \sum_{j_{2}<}^{\prime \prime} \ldots \\
& \times \sum_{\cdots<j_{m-1}}^{\prime \prime} \sigma_{\mathbf{r}_{j_{1}}}^{-} \cdots \sigma_{\mathbf{r}_{j_{m-1}}^{-}}^{-} \sigma_{\mathbf{p}}^{-}|F\rangle,
\end{aligned}
$$

where the double prime restricts the sums to vectors $\mathbf{r}_{\mathbf{j}}$ $\neq \mathbf{r}, \mathbf{p}$. Thus, for $\mathbf{r} \neq \mathbf{p}$ there are $\left(\begin{array}{c}N-2 \\ m-1\end{array}\right)$ terms contributing equally to $\left\langle\psi_{m}\left|\hat{n}_{\mathbf{r}}\right| \Lambda\right\rangle$. Hence, by taking into account the wave function constraint $\Sigma_{\mathbf{r}} \varphi_{\lambda}(\mathbf{r}) \equiv 0$ so as to ensure a total spin $S=N / 2-1 \quad(\lambda>0)$, we obtain

$$
\begin{aligned}
\left\langle\psi_{m}\left|\hat{n}_{\mathbf{r}}\right| \Lambda\right\rangle & =(m-1) ! m !\left(\begin{array}{c}
N-2 \\
m-1
\end{array}\right) \alpha_{m} \beta_{m} \sum_{\mathbf{p}} \varphi_{\lambda}(\mathbf{p})\left(1-\delta_{\mathbf{r}, \mathbf{p}}\right) \\
& =-\sqrt{\frac{m(N-m)}{N(N-1)}} \varphi_{\lambda}(\mathbf{r}),
\end{aligned}
$$

where $\alpha_{m}$ is taken as in Eq. (9). Therefore the particleparticle correlations of Eq. (16) are finally given by

$$
C_{\mathbf{r}, \mathbf{r}^{\prime}}(t)=\frac{N \rho(1-\rho)}{N-1} \sum_{\text {single } \lambda \text { levels }} e^{-\lambda t} \varphi_{\lambda}(\mathbf{r}) \varphi_{\lambda}^{*}\left(\mathbf{r}^{\prime}\right),
$$

which at most requires the evaluation of single spin-wave (Bloch) excitations in disordered ferromagnetic backgrounds. This is the main result of this section. Certainly, the usefulness of the quantum spin operational formalism developed so far is subject to the knowledge of such excitations. However, much is known about their properties and density of states $[11,15]$, and in fact this enables us to obtain explicit results, particularly for large time asymptotic regimes (Sec. III). A similar reasoning for non-instantaneous $\nu$-point correlators would involve $|\Lambda\rangle$ states with total spin $S=N / 2$ $-\mathcal{M}$, i.e., states with $\mathcal{M}$-interacting magnons $(\mathcal{M}$ $=1, \ldots, \nu)$. In this more general case however, analytical progress seems difficult given the scarcity of exact results in $d>1$, even for ordered substrates [16].

\section{AUTOCORRELATION FUNCTIONS OF DILUTED LATTICES}

A subcase of Eq. (24) entailing particular interest and which is related to the probability of hard-core random walkers returning to the origin, is that of autocorrelation functions $C(t)$. This corresponds to averaging Eq. (24) over all locations $\mathbf{r}=\mathbf{r}^{\prime}$. For isotropic structures, it is plausible that the number of sites a random walker (particle) has visited after a time $t$ is proportional to the volume $R^{d}(t)$, where $R$ is the root mean square displacement in a substrate of dimensionality $d$ (either Euclidean or fractal). Thus for densities $\rho$ $\ll 1 / R^{d}$, the probability of returning to the origin should in principle scale as $C(t) \propto R^{-d}(t)$. Departures from normal or Fickian diffusion $\left(R \propto t^{1 / 2}\right)$ in disordered lattices can therefore be studied by both, (i) evaluating the asymptotic behavior of autocorrelations and (ii) averaging the hopping distribution.

Autocorrelations are in turn closely related to the density of states in the substrate. In fact, due to the normalization of the single level eigenfunctions, after averaging over the sites (origins) of a given sample, in the large $N$ limit clearly Eq. (24) reduces to

$$
C(t)=\frac{A_{\rho}}{N} \sum_{\text {single } \lambda \text { levels }} e^{-\lambda t},
$$

where $A_{\rho} \equiv \rho(1-\rho)$. So, the form of the density of states for $\lambda \rightarrow 0$ determines entirely the large time asymptotic behavior of the autocorrelation function for hard-core particles at finite densities.

Before continuing and in preparation for the analysis of autocorrelations in disordered scenarios, it is worthwhile to pause and consider the finite size scaling regimes emerging asymptotically from Eq. (25) in regular ordered situations $\left(\left\{J_{\left\langle\mathbf{r r}^{\prime}\right\rangle}\right\} \equiv J\right)$. Let us consider briefly a $d$-dimensional hypercubic lattice with $N=L^{d}$ sites and periodic boundary conditions. It is well known that the corresponding $\lambda$ levels are $2 J \sum_{j=1}^{d}\left[1-\cos \left(2 \pi n_{j} / L\right)\right]$ with $n_{j}=0, \ldots, L-1$. From Eq. (25) it is straightforward to check that the autocorrelation function of such finite system factorizes as

$$
C(t)=A_{\rho} \frac{e^{-2 d J t}}{L^{d}}\left[\left(\sum_{n=0}^{L-1} e^{2 J t \cos (2 \pi n / L)}\right)^{d}-1\right]
$$

where the last term takes care of the cancellation of the $\lambda$ $=0$ contribution. Thus, it can be readily verified that there is a scaling regime $L \rightarrow \infty, J t \rightarrow \infty$ for which the autocorrelation scales with a universal function $\mathcal{F}(\tau)$ so long as $\tau \equiv J t / L^{2}$ is held constant, namely,

$$
\begin{gathered}
C(L, t)=(J t)^{-d / 2} \mathcal{F}(\tau), \\
\mathcal{F}(\tau)=A_{\rho} \tau^{d / 2}\left[\vartheta_{3}^{d}(q)-1\right], \quad \vartheta_{3}(q) \equiv 1+2 \sum_{n=1}^{\infty} q^{n^{2}},
\end{gathered}
$$

where $q=e^{-4 \pi^{2} \tau}$ and $\vartheta_{3}(q)$ is a Jacobi theta function of the third kind [17]. Hence, the typical size of the system sets the time scales $\left(t \propto L^{2}\right)$ for which the dynamics becomes diffusive. Also, recalling that $\lim _{\tau \rightarrow 0} \sqrt{\tau} \vartheta_{3}(q)=1 / 2 \sqrt{\pi}$, the autocorrelations of the infinite system result in long time diffusive tails (as they should), whose asymptotic kinetics is given by

$$
C(t) \sim \frac{A_{\rho}}{(4 \pi)^{d / 2}}(J t)^{-d / 2} .
$$


Turning to more general situations, a case of interest is that of bond-(hopping-) diluted lattices. More specifically, we will focus attention on the following binary hopping probability distribution

$$
P\left(J^{\prime}\right)=p \delta_{J, J^{\prime}}+(1-p) \delta_{J^{\prime}, 0}
$$

defined uniformly throughout all lattice bonds. For shortrange hoppings, say, e.g., nearest neighbors, a purely geometric effect, known as the percolation transition, arises from the configurational aspects caused by dilution. We address the reader to Ref. [18] for a detailed introduction to this subject which has been found useful to characterize a rich variety of diluted systems including spin systems [19]. Here, we just mention that there exists a critical concentration $p_{c}$ below which only finite clusters exist and above which a cluster spanning the (infinite) lattice is formed.

Of course in $d=1$ any bond removal disconnects the lattice, so $p_{c}=1$. However, as we shall see in Sec. III A, as in the regular case [Eq. (27)], autocorrelations also exhibit an asymptotic scaling regime for finite dilutions but through a dilution-dependent scale of length. In higher Euclidean dimensions, extensive research $[11,15,18,20]$ has led to the conclusion that percolation clusters are statistically selfsimilar at the transition, and so are random fractals. They are characterized by various scaling dimensions (fractal, spectral) for the various processes (mass, density of states) measurable on the fractal. Thus, exploiting scaling results for the spectral dimension, in Sec. III B we shall discuss the implications which the resulting density of states imposes on the large time average behavior of Eq. (25).

\section{A. Diluted chain}

In the one-dimensional case for $0<p<1$ the distribution (29) breaks the $N$-site chains into a collection of finite disconnected segments, each having a number $1 \leqslant L \leqslant N-1$ of consecutive bonds, or $L+1$ correlated sites. So, the probability $W(L)$ to find a chain with $L$ consecutive nonvanishing transition rates and free boundaries is independent of the chain location and is given by

$$
W(L)=\left\{\begin{array}{l}
(1-p)^{2} p^{L} \text { if } 1 \leqslant L \leqslant N-2, \\
(1-p) p^{L} \text { if } L=N-1, \\
p^{L} \text { if } L=N \quad \text { (periodic chain). }
\end{array}\right.
$$

On the other hand, as shown in Sec. II, for a given segment of $(L-1)$ bonds, it is sufficient to consider the corresponding evolution operator $H_{L}$ within the single spin excitation sector. From Eq. (8), it can be readily checked that $H_{L}$ can be recast in terms of the following $L \times L$ tridiagonal form

$$
H_{L}=2 J\left[\begin{array}{ccccc}
1 & -1 & 0 & \cdots & 0 \\
-1 & 2 & -1 & \ddots & \vdots \\
0 & \ddots & \ddots & \ddots & 0 \\
\vdots & \ddots & -1 & 2 & -1 \\
0 & \cdots & 0 & -1 & 1
\end{array}\right] \text {, }
$$

with eigenvalues

$$
\lambda_{L}(n)=4 J\left(1-\cos \frac{n \pi}{L}\right), \quad n=0,1, \cdots, L-1 .
$$

Assuming a constant particle density $\rho$ per chain, the overall autocorrelations must be averaged over both histories (time) and samples (hopping disorder). Thus, the autocorrelations arising from all possible distributions of disconnected segments reads

$$
\langle C(t)\rangle=A_{\rho} \sum_{L=1}^{N-1} W(L) \sum_{n=1}^{L} e^{-\lambda_{L+1}(n) t},
$$

where $A_{\rho}$ is taken as in Eq. (25). Here the first sum, denoted by the brackets on the left hand side, runs over segments of $L$ consecutive bonds and takes into account the hopping disorder, whereas the second sum runs over eigenvalue levels $\lambda_{L+1}(n)>0,1 \leqslant n \leqslant L$, and carries out the time dependent average. We are especially interested to elucidate the asymptotic behavior of Eq. (33) for arbitrarily large times and finite dilution regimes $0<p<1$. To this aim, it is useful to introduce the natural length scale emerging in the problem, namely, the percolation correlation length

$$
\xi=-\frac{1}{\ln p}, \quad 0<p<1
$$

which measures the effective distance between missing hopping rates. On general grounds, this scale can be regarded as the mean distance between two sites belonging to the same cluster which, in higher dimensions, diverges as $\xi \sim(p$ $\left.-p_{c}\right)^{-\nu}$ with a universal exponent $\nu$ depending solely on the space dimensionality [18].

Now, from Eqs. (30) and (33) it follows that in the thermodynamic limit $N \rightarrow \infty$ we are left with the calculation of

$$
\langle C(t)\rangle=A_{\rho} \frac{(1-p)^{2}}{p} \sum_{L=2}^{\infty} \sum_{n=1}^{L} e^{-\left[L / \xi+\lambda_{L}(n) t\right]} .
$$

Since $\lambda_{L}(n)=2 J(n \pi / L)^{2}+\mathcal{O}\left(1 / L^{4}\right)$ it is clear that the dominant contributions to Eq. (35) when $t \rightarrow \infty$, are basically contained in large $L$ segments. Introducing the (dimensionless) scaling variables

$$
\tau=\frac{J t}{\xi^{2}}, \quad s=\frac{L}{\xi}
$$

it is straightforward to verify that within the scaling regime $\xi \gg 1$ with $J t \gg 1$ Eq. (35) can be written as

$$
\langle C(\tau)\rangle \sim \frac{A_{\rho}}{\xi} \int_{0}^{\infty} e^{-s} \sum_{n=1}^{s \xi} e^{-2 \pi^{2} n^{2} \tau / s^{2}} d s .
$$

For $\tau \ll 1$, the sum over $n$ can be replaced by an integral, and a result $\langle C(t)\rangle \propto t^{-1 / 2}$ (of pure-system form) follows. This is the asymptotic behavior on one side of a crossover occurring at $\tau \sim 1$. The other side has the more interesting (disorderdominated) asymptotic behavior, obtained by considering $\tau$ $\gg 1$. Then the sum is dominated by the $n=1$ term, so 


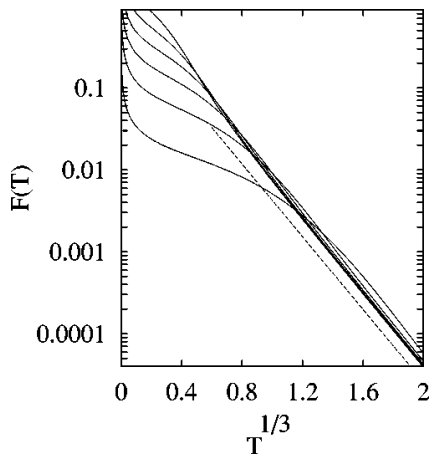

FIG. 1. Stretched exponential decay and scaling regime for autocorrelation functions of diluted chains. $\tau$ (here denoted as $T$ ), is the dimensionless variable of Eq. (36) involving the time $t$ divided by the square of the percolation correlation length $\xi$. Following Eq. (39) in the text, $F(\tau)$ is here taken as $\langle C(\xi, \tau)\rangle /\left[\xi^{-1} \tau^{1 / 6} \sqrt{2 a \pi / 3}\right]$. Solid lines denote different degrees of dilution $p=e^{-1 / \xi}$. The uppermost curve at the left corresponds to $p=0.6$, and the lower curves to $p=0.5,0.4,0.3,0.2$, and 0.1 , in descending order. The slope of the dashed line is $-\frac{3}{2}(2 \pi)^{2 / 3} \log _{10} e$.

$$
\langle C(\tau)\rangle \sim \frac{A_{\rho}}{\xi} \int_{0}^{\infty} e^{-\left[s+\left(2 \pi^{2} / s^{2}\right) \tau\right]} d s .
$$

To obtain the asymptotic behavior of the integral we use a saddle-point expansion around the minimum of the exponent, at $s_{0}=\left(4 \pi^{2} \tau\right)^{1 / 3}$, which becomes exact in the scaling limit $\tau \gg 1$. After elementary manipulations, this finally yields autocorrelations characterized by a universal scaling function $\mathcal{U}(\tau)$ exhibiting a stretched exponential decay together with a subdominant power law prefactor. More explicitly, for $\tau \gg 1, \xi \gg 1$,

$$
\begin{gathered}
\langle C(\xi, \tau)\rangle=\xi^{-1} \mathcal{U}(\tau), \\
\mathcal{U}(\tau)=A_{\rho} \sqrt{\frac{2 a \pi}{3}} \tau^{1 / 6} \exp \left(-\frac{3 a}{2} \tau^{1 / 3}\right),
\end{gathered}
$$

where $a=(2 \pi)^{2 / 3}$. This is the central result of this subsection, and it corresponds to localization when the average cluster size is smaller than the pure diffusion length.

To check the reliability of this form we address the reader's attention to Fig. 1 in which autocorrelations are computed directly from Eq. (35). The data collapse obtained for different dilutions clearly confirm Eq. (39) and provides further evidence for the existence of an asymptotic scaling regime characterized by the variable $\tau=J t / \xi^{2}$ and showing the crossover at $\tau \sim 1$ between pure system diffusive behavior $(\tau<1)$ and localization $(\tau>1)$. Notice that so long as $p$ $\neq 1$, no matter how small the dilution is, it induces at sufficiently large time a different dynamics from that developed by the regular, purely diffusive case [Eq. (28)]. This contrasting behavior comes from the interplay of a pure diffusion length $\left(\propto t^{1 / 2}\right)$ and the percolation length $\xi$ (roughly the average segment length) which diverges at the threshold $p$ $=1$.

\section{B. Percolation cluster}

In higher dimensions the dynamic behavior is much richer by virtue of (i) the more interesting fractal structure of the percolation geometry at the threshold $p_{c}$ and (ii) the exis- tence of the infinite (spanning) cluster above $p_{c}$. We therefore have to distinguish finite cluster contributions to $\langle C(t)\rangle$ from the infinite cluster contribution when $p \geqslant p_{c}$.

We first consider how such features might affect the Goldstone argument [21]. The spin symmetry properties remain as for the pure system. The full rotational symmetry by an angle $\alpha$ around an arbitrary spin direction $\hat{\mathbf{n}}$, evidently leaves the evolution operator (8) invariant, i.e., $H$ $=e^{-i \alpha \mathbf{S} \cdot \hat{\mathbf{n}}} H e^{i \alpha \mathbf{S} \cdot \hat{\mathbf{n}}}$. However, the steady (ground) states of Eq. (9) do not preserve such symmetry $\forall \hat{\mathbf{n}} \neq \hat{z}$. Because of this spontaneous symmetry breaking, in the thermodynamic limit $N \rightarrow \infty$ a low-lying band of gapless or Goldstone modes can be expected in the pure system [21] and, because of its unlimited size, also on the infinite cluster $\left(p \geqslant p_{c}\right)$ irrespective of the hopping disorder in $H$. Moreover, the Goldstone modes are the $|\Lambda\rangle$ states already introduced in Eq. (19) and those with large characteristic scale are ultimately responsible for the emergence of a slow asymptotic kinetics [Eqs. (26), (27)].

Next consider the finite clusters (for $p \neq p_{c}$ ). They are distributed with size distribution having characteristic scale $\xi$ but nevertheless extending with exponential tail to infinite size (see also Sec. IV). Without these tails the finite size cutoff would introduce a gap in the spectrum of the (Goldstone) modes on the ensemble of finite clusters.

The contributions, to dynamic properties such as $\langle C(\xi, t)\rangle$, from excitations on finite clusters and on the infinite cluster (for the case $p \geqslant p_{c}$ ) can be obtained from the density of single levels $\omega(\lambda)$ or density of states (DOS) involved in Eq. (25). Therefore, the averaged autocorrelations can be written as

$$
\langle C(t)\rangle=A_{\rho} \int_{0}^{\infty} \omega(\lambda) e^{-\lambda t} d \lambda .
$$

The specific form of the DOS for $\lambda \rightarrow 0$ from modes on the finite clusters is closely related to the role played by Lifshitz tails $\sim e^{-\lambda^{-r}}(r>0)$ in the energy distribution, a typical issue occurring in the presence of disorder [22]. Consider, for example, a square lattice of which a fraction $p$ of bonds are accessible. Below the percolation threshold the low energy DOS is characterized by Lifshitz tails which are contributed by large regions of connected sites. In the onedimensional case (Sec. III A) strings of length $L$ occur with probability $p^{L}$ and contribute low-lying ferromagnetic modes with $\lambda \propto L^{-2}$, so the DOS form is $\omega(\lambda) \propto p^{\lambda^{-1 / 2}}$. In fact, such a tail in the DOS is dominant in determining the relaxation dynamics referred to in Sec. III A.

Above the percolation threshold Lifshitz tail modes are also present, but are swamped by the power law tail $\omega(\lambda)$ $\sim \lambda^{d / 2-1}$ due to low energy excitations on the infinite cluster. Thus for $p>p_{c}$ we expect the usual diffusive decay $\langle C(t)\rangle$ $\propto t^{-d / 2}$ at long times. In passing, it is instructive to check the case $p=1$. It is well known that for an hypercubic lattice the DOS of large wavelength excitations behaves as $\omega(\lambda)$ $\sim\left[\Omega_{d} / 2(2 \pi)^{d}\right] \lambda^{d / 2-1}$, where $\Omega_{d}$ is the surface of the $d$-dimensional unit sphere. Thus after integrating Eq. (40), we recover precisely both the asymptotic diffusive tails and amplitudes already obtained in Eq. (28).

At $p=p_{c}$, the Lifshitz tail is again present in $\omega(\lambda)$ and swamped by a power tail which in this case has a nontrivial 
exponent $d_{s}-1$ where $d_{s}$ is, by definition, the spectral dimension and is the ratio of the fractal dimension $d_{f}$ and the dynamic exponent $z$ (which are respectively the length scaling dimensions for mass and frequency): $d_{s}=d_{f} / z$. These exponents have been investigated for the linear spin wave problem at the percolation threshold, by direct numerical calculation [23] and via scaling relations $[11,15] d_{f}=d$ $-\beta / \nu, z=2+(t-\beta) / \nu$ to the exponents for percolation conductance $(t)$, correlation length $(\nu)$, and density $(\beta)$. For all $d>1, d_{s}$ is very close to (though not exactly equal to) the conjectured Alexander-Orbach [24] value 2/3 (a factor of 2 arises here because the basic equation for ferromagnetic spin waves involves frequency squared).

Hence from Eq. (40) we immediately obtain for the exact finite-density autocorrelation function of hard-core diffusing particles at large times at the percolation threshold $\left(p=p_{c}\right)$

$$
\langle C(t)\rangle \propto t^{-d_{s},}
$$

where $d_{s}=(d \nu-\beta) /(2 \nu+t-\beta)$ is very close to $2 / 3$ for all $d>1$.

This is in fact the $\left(t / \xi^{z}\right) \rightarrow 0$ scaling limit of the following general form implied by scaling considerations applicable when both $t$ and $\xi$ are large:

$$
\langle C(\xi, t)\rangle \propto t^{-d_{s}} f\left(t / \xi^{z}\right) .
$$

This form includes the crossover at $t \sim \xi^{z}$, and leads us to conjecture that at $p_{c}$ within the percolation cluster, the diffusive behavior is characterized by an anomalous root mean square displacement $R \propto t^{1 / z}, z \sim 3 d_{f} / 2$ where $d_{f}$ is the fractal dimension $d-\beta \nu$. Equations (41) and (42) are the main results of this subsection.

\section{CONCLUSIONS}

The mapping to quantum spin systems exploited in this paper gives a powerful approach to the stochastic dynamics of hard-core particle systems. Using it we have been able to obtain in Sec. II exact dynamical properties (including the space and time dependent pair correlation function) for the interacting system at any density in terms of corresponding properties for linearized spin wave dynamics. The procedure applies equally well to disordered systems, and in addition Goldstone arguments still apply there. In the applications made here to disordered systems we have chosen to emphasize the diluted case, because of the dramatic effects seen there by virtue of the underlying percolation geometry.

A somewhat surprising result is that hard-core particle diffusion on diluted chains is quite interesting, any dilution disconnects the chain, so the static diffusion constant strictly vanishes. Yet finite-time dynamics is nontrivial, exhibiting scaling behavior and stretched exponential autocorrelation functions in the time domain (Sec. III A). The scaling behavior is analogous to that calculated earlier for the apparently much simpler (but equivalent) problem of ferromagnetic spin wave dynamics of diluted chains [25]. There the calculations were carried out in the frequency-wave vector domain, for comparison with inelastic neutron scattering measurements, so the stretched exponential time behavior was not identified, but it should also be present there.

For interacting particle dynamics on higher dimensional diluted lattices, by virtue of the mapping we have been able to draw on scaling procedures and results for spin wave dynamics of diluted systems. This is particularly direct for the autocorrelation function of the diffusing hard-core particle system, which is given completely in terms of a DOS which is the same as for spin wave dynamics.

Below the percolation threshold the diffusion is entirely on finite clusters. The low frequency density of states determining the long time behavior comes from large clusters whose occurrence probability is exponentially small in cluster size, very much as in the one dimensional case. Above the percolation threshold the finite cluster contribution is similar, but it is now swamped by the infinite (spanning) cluster contribution which, well away from the threshold gives a behavior like that for the pure system. At and near the threshold $\left(p \sim p_{c}\right)$ the diverging scale of the percolation geometry induces scaling behavior for the long time dynamics. Here the DOS has an anomalous power law dependence on frequency $\lambda$; the associated exponent has been obtained via the relationship of the DOS to that for the linear spin wave system and further relationships to percolation processes including (static linear) conductance; the exponent in the DOS power law is given in terms of $t, \beta, \nu$, the exponents dependence on $\left|p-p_{c}\right|$ of percolation conductance, density and correlation length. The consequent exponent in the power law time decay of the autocorrelation function at $p_{c}$ turns out to be close to $2 / 3$ for any $d>1$ (in accord with the approximate Alexander-Orbach conjecture [24]). The scaling behavior of the space and time dependent correlation function depends on the DOS exponent and also on the length scaling exponent for the frequency $\lambda$. This so called dynamic critical exponent is also related to $t, \beta \nu$, and hence we can give the power law dependence of the characteristic diffusion length on time. This generalization also allows discussion of the crossover due to the competition between characteristic diffusion length and percolation correlation length near the transition.

Clearly, further extensions are desirable. For the dilute systems the most obvious ones are the calculation of space and time dependent correlation and crossover functions, and also probability distributions rather than just averages. In addition simulation confirmation of the analytic predictions or experimental comparisons would be desirable. As regards more general disorder, other types of substitutional disorder could clearly be treated by the methods we have used. A more challenging extension is to the biased diffusion of hardcore particles [26]. Much has been done exploiting the quantum spin mapping for the biased case along with adsorptiondesorption processes [6]. But biased hard-core particle diffusion on disordered lattices is at present beyond the exact techniques exploited here.

\section{ACKNOWLEDGMENTS}

We would like to thank D. C. Cabra for valuable discussions and remarks. M.D.G. acknowledges financial support from CONICET, Argentina. The research of R.B.S. was partly supported by the EPSRC under the Oxford Condensed Matter Theory Rolling Grant No. GR/M 04426. 
[1] Nonequilibrium Statistical Mechanics in One Dimension, edited by V. Privman (Cambridge University Press, Cambridge, 1996).

[2] J. P. Bouchaud and A. Georges, Phys. Rep. 195, 127 (1990).

[3] S. Havlin and D. ben-Avraham, Adv. Phys. 36, 695 (1987); J. W. Haus and K. W. Kehr, Phys. Rep. 150, 263 (1987).

[4] N. G. van Kampen, Stochastic Processes in Physics and Chemistry, 2nd ed. (North Holland, Amsterdam, 1992).

[5] This strategy which can be traced back over more than two decades. For a comprehensive review and literature list consult D. C. Mattis and M. L. Glasser, Rev. Mod. Phys. 70, 979 (1998).

[6] M. D. Grynberg and R. B. Stinchcombe, Phys. Rev. Lett. 76, 851 (1996); ibid. 74, 1242 (1995).

[7] S. Alexander, J. Bernasconi, W. R. Schneider, and R. Orbach, Rev. Mod. Phys. 53, 175 (1981).

[8] S. Alexander and T. Holstein, Phys. Rev. B 18, 301 (1978).

[9] L.-H. Gwa and H. Spohn, Phys. Rev. Lett. 68, 725 (1992); F. C. Alcaraz, M. Droz, M. Henkel, and V. Ritenberg, Ann. Phys. (N.Y.) 230, 250 (1994); G. M. Schütz, Phys. Rev. E 53, 1475 (1996); M. Henkel, E. Orlandini, and G. M. Schütz, J. Phys. A 28, 6335 (1995); M. Barma, M. D. Grynberg, and R. B. Stinchcombe, Phys. Rev. Lett. 70, 1033 (1993).

[10] R. B. Stinchcombe, M. D. Grynberg, and M. Barma, Phys. Rev. E 47, 4018 (1993).

[11] See articles by A. Aharony, R. Orbach, H. E. Stanley, and R. B. Stinchcombe, in Scaling Phenomena in Disordered Systems, edited by R. Pynn and A. Skjeltorp (Plenum, New York, 1985).

[12] K. Kawasaki, in Phase Transitions and Critical Phenomena, edited by C. Domb and M.S. Green (Academic Press, London, 1972), Vol. 2.

[13] For a thorough account of these topics within the perspective of linear response theory, see R. Kubo, M. Toda, and N. Hashitsume, Statistical Physics II: Nonequilibrium Statistical Mechanics, 2nd ed. (Springer-Verlag, Berlin, 1991).

[14] For finite lattices $\Delta t \propto 1 / N$, thus late asymptotic regimes require $n \gg N$.

[15] A vast body of work on these issues can be consulted in IllCondensed Matter, Les Houches, Session XXXI, edited by R. Balian, R. Maynard, and G. Tolouse (North-Holland, Amsterdam, 1979).

[16] However, for $\nu=2$, see M. Wortis, Phys. Rev. 132, 85 (1963).

[17] Handbook of Mathematical Functions, edited by M. Abramowitz and I.A. Stegun (Dover, New York, 1958); I.S. Gradshteyn and I.M. Ryzhik, Table of Integrals, Series and Products, 5th ed. (Academic Press, New York, 1994).

[18] D. Stauffer and A. Aharony, Introduction to Percolation Theory, 2nd ed. (Taylor \& Francis, New York, 1994).

[19] R. B. Stinchcombe, Diluted Magnetism in Phase Transitions and Critical Phenomena, edited by C. Domb and J.L. Lebowitz (Academic Press, London, 1983), Vol. 7.

[20] B. B. Mandelbrot and J. A. Given, Phys. Rev. Lett. 52, 1853 (1984).

[21] J. Goldstone, Nuovo Cimento 19, 155 (1961); Y. Nambu, Phys. Rev. Lett. 4, 380 (1960).

[22] J. P. Clerc, G. Giraud, J. M. Laugier, and J. M. Luck, Adv. Phys. 39, 191 (1990).

[23] S. J. Lewis and R. B. Stinchcombe, Phys. Rev. Lett. 52, 1021 (1984).

[24] S. Alexander and R. Orbach, J. Phys. (France) Lett. 43, L625 (1982).

[25] A. C. Maggs and R. B. Stinchcombe, J. Phys. A 17, 1555 (1984).

[26] For a recent account on this and related processes see B. Derrida and M. R. Evans in Ref. [1]. 